\title{
LOS CAMINOS CEREMONIALES EN LOS APUS DEL TAWANTINSUYU ${ }^{1}$
}

\author{
CEREMONIAL ROADS IN THE APUS OF TAWANTINSUYU
}

\author{
Christian Vitry ${ }^{2}$
}

\begin{abstract}
En el proceso de expansión territorial de los Incas, los sistemas viales jugaron un rol estructural fundamental, llegando a conformar -a través de la infraestructura edilicia- una suerte de continuidad espacial desde la capital del Tawantinsuyu hasta las lejanas periferias. Los vastos paisajes cordilleranos, con sus altas montañas, lejos de ser considerados como obstáculos o lugares inaccesibles, fueron la morada de deidades, conformando una territorialidad simbólica e ideológica con una fuerte presencia material y cultural en los paisajes. Hasta el presente se tiene conocimiento de poco más de doscientas montañas con evidencias arqueológicas en los Andes y cordilleras vecinas, en quince de estas montañas se realizaron ofrendas humanas y en muchas de ellas se registraron caminos ceremoniales que conducen a lo alto de los picos. En otras oportunidades hicimos referencia a los caminos del nevado de Chañi y el volcán Llullaillaco. En la presente investigación daremos a conocer un amplio listado de montañas que poseen caminos, intentando establecer algunos indicadores arqueológicos y espaciales de estas particulares vías que fueron desarrolladas en unos de los ambientes más extremos del mundo.
\end{abstract}

Palabras claves: caminos ceremoniales, apus, arqueología de alta montaña, capacocha, Montañas sagradas.

During the process of territorial expansion of the Incas, the road systems played an essential structural role by shaping a type of spatial continuity through built infrastructure, which started in the capital of Tawantinsuyu and spread to distant peripheries. Far from being considered as obstacles or inaccessible places, the vast Andean landscapes and their high mountains were the abode of deities, which shaped a symbolic and ideological territoriality with strong material and cultural presence in the landscapes. So far, a little more than two hundred mountains with archaeological evidence have been studied in the Andes and other neighboring mountain ranges. On fifteen of these mountains human offerings were made, and in many of them, ceremonial roads leading to the top of the peaks have been surveyed. We have previously referred to the paths of Mount Chañi and the Llullaillaco volcano. Here, we present an extensive list of mountains with roads in an attempt to establish some archaeological and spatial indicators of these particular paths, built in some of the most extreme environments in the world.

Key words: Ceremonial roads, apus, high mountain archaeology, capacocha, sacred mountains.

Durante el siglo XV y parte del XVI, la Cordillera de los Andes y sus alrededores inmediatos -hacia la costa desértica del Pacífico y el piedemonte selvático oriental- fue el escenario donde floreció y se expandió el Tawantinsuyu, una propuesta política generada por los Incas (Lumbreras 2006), quienes supieron aprovechar inteligentemente una suerte de "vacío" que había quedado en la región pocos siglos atrás con la ausencia de Tiahuanaco (Meyers 2002:533).

En este contexto, la política expansiva del Tawantinsuyu, concretó un dominio territorial que se extendió, desde el sur de Colombia hasta el sur de Santiago de Chile y el norte de la provincia de Mendoza en Argentina, ocupando una superficie aproximada de dos millones de kilómetros cuadrados.

La conquista territorial incaica no solo fue de índole política-económica, sino también simbólicareligiosa; tampoco fue exclusivamente "horizontal", sino que tuvo un componente "vertical" muy importante, pues hasta la fecha hemos registrado 226 montañas que fueron ascendidas y resignificadas, y sobre ellas se construyeron recintos ceremoniales y

\footnotetext{
${ }^{1}$ Una primera versión de este trabajo fue presentada en el Qhapaq Ñan II, Taller Internacional en torno al Sistema Vial Inkaico (24-27 de octubre de 2017), efectuado en Salta, Argentina. Este manuscrito fue evaluado por pares externos y editado por el Comité Editorial de Chungara.

2 Universidad Nacional de Salta. Programa Qhapaq Nan, Subsecretaría de Patrimonio de la Provincia de Salta, Salta, Argentina.chvitry@yahoo.com; vitrydibellochristian@hum.unsa.edu.ar
}

Recibido: abril 2019. Aceptado: abril 2020. 
caminos, y también en algunas se realizaron sacrificios humanos Beorchia Nigris 1985 y 2001; Besom 2009; Ceruti 2003; Reinhard y Ceruti 2000; Schobinger 1998; Vitry 1997, 2008, 2017; entre otros). Esta geografía sagrada, jalonada por centenares de geosímbolos como montañas y huacas de diversa índole, posee una particular distribución y concentración hacia el Sur de Cusco (Vitry 2017:36) (Figura 1).

La inscripción simbólica sobre el paisaje fue objeto de numerosos estudios realizados por Bauer, Zuidema,

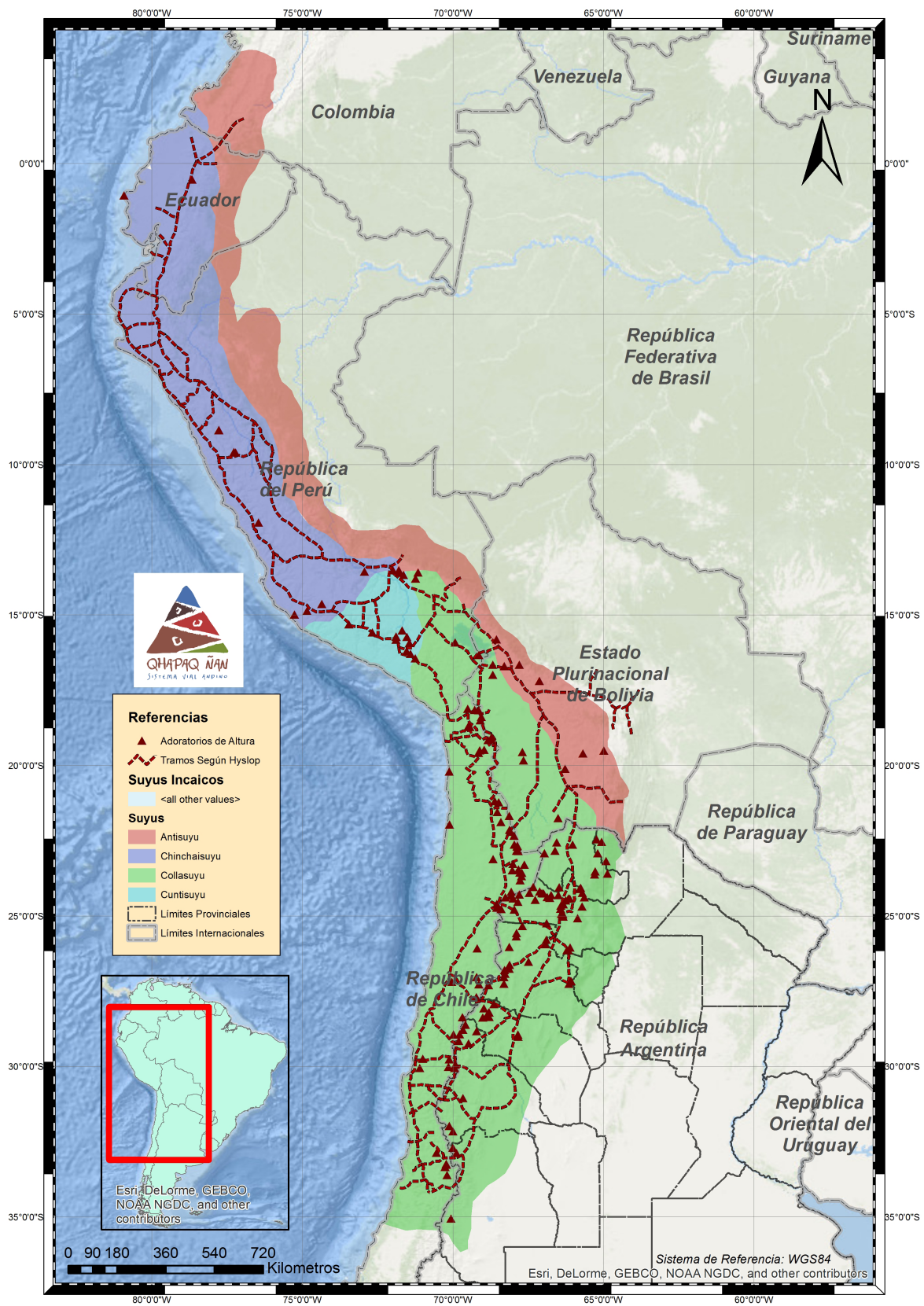

Figura 1. Distribución espacial de los adoratorios de altura en la cordillera.

Spatial distribution of height shrines in the mountain range. 
Sherbondy, Chávez Bayón, Rowe, Aveni, Dearborn, Schreiber y Urton, entre otros, que se dedicaron a interpretaciones espaciales de los ceques vinculados con la organización social de los Incas (cf. Bauer 2000). Por otra parte, los restos arqueológicos de estas montañas sacralizadas fueron estudiadas desde la década de 1950, particularmente con el hallazgo del niño del Cerro El Plomo en Chile y posteriores hallazgos en Argentina como los del Cerro El Toro (San Juan) en 1963 y el Aconcagua en 1985 (Beorchia Nigris 1985, 2001; Schobinger 1986).

\section{Caminos Ceremoniales en Montañas Sagradas}

Tanto la arqueología de alta montaña como los estudios de los sistemas viales incas tuvieron un significativo crecimiento en las últimas dos décadas, sin embargo, los caminos ceremoniales que ascienden a las montañas con sitios arqueológicos poseen escasos antecedentes; en tal sentido, tenemos los trabajos de Rebitsch (1966); Niemeyer y Rivera (1983); Núñez Henríquez (1981); Reinhard (1983 y 1997); Schobinger (1986); Beorchia Nigris (1985); Hyslop (1992); Lynch (1996); Astuhuamán Gonzáles (1999); Ceruti (2003); Castro et al. (2004); entre otros, quienes los mencionaron pero no los hicieron objeto de estudios sistemáticos específicos.

En los últimos años las investigaciones de los adoratorios de altura empezaron a integrar al sistema vial Inca de una manera más detallada y contextual, abordando el estudio de los caminos ceremoniales de las altas montañas y geosímbolos (Bonnemaison 1992) como elementos de importancia para la comprensión de la geografía sagrada y el paisaje cultural andino (Cruz 2009; Cruz et al. 2013; Ibacache y Cantarutti 2006; Ibacache et al. 2016; Jacob y Leibowicz 2011; entre otros). Dentro de esta línea hemos realizado investigaciones en los caminos ceremoniales del Volcán Llullaillaco y el Nevado de Chañi (Vitry 2007, 2009, 2015, 2017), guiados por una serie de preguntas orientadoras de la investigación:

¿hay algún patrón constructivo que diferencie los caminos rituales de los comunes?, ¿cuáles son los elementos o estructuras asociadas a los caminos rituales?, ¿tienen las montañas que poseen caminos alguna característica en común?, ¿todas las montañas con ofrendas humanas poseen caminos?, ¿los caminos ceremoniales de las montañas guardan relación con los caminos troncales y/o con las comunidades próximas? (Vitry 2007:70).

Tras varios años de recorrer la cordillera creemos estar en condiciones de responder, con diferente grado de profundidad, gran parte de los cuestionamientos relacionados con los caminos ceremoniales. Para ello, recurrimos a modo de síntesis a una serie de indicadores arqueológicos que nos ayudarán a organizar la información.

\section{Indicadores arqueológicos de los caminos y sitios ceremoniales de alta montaña}

Presentamos una serie de indicadores arqueológicos, basados en la evidencia material de una muestra bastante representativa de caminos ceremoniales que ascienden a las altas cumbres andinas y que resultan factibles de reconocimiento en el terreno y de ser estudiados arqueológicamente (Tabla 1). Esta lista dista mucho de ser completa y en ella se incorporaron solamente los casos comprobados por fuentes publicadas o referencias inéditas brindadas por profesionales arqueólogos. Una gran cantidad de referencias quedaron fuera, pues es mejor actuar con cautela e ir sumándolas a medida que se avance con las investigaciones de estos caminos tan conspicuos.

\section{Caminos formalmente construidos}

Por lo general, las estructuras arqueológicas que se localizan en las montañas suelen estar bastante bien conservadas, debido fundamentalmente a la escasa influencia de los factores antrópicos y naturales (salvo excepciones puntuales). Sin embargo, los caminos no corren la misma suerte, ya que poseen una gran extensión longitudinal y se localizan en laderas inestables, formadas por rocas sueltas, sufriendo además los procesos erosivos ocasionados por la acumulación de nieve. No obstante, los segmentos que lograron conservarse hasta el presente han mostrado que no se trata de una huella hecha por el uso, sino más bien de una construcción formal e intencionada, tal como veremos a continuación en los demás indicadores arqueológicos.

\section{Anchos constantes de los caminos}

Los caminos ceremoniales registrados hasta el momento nos dan cuenta de que poseen un ancho constante durante gran parte del ascenso, dependiendo esto de las características del terreno y relieve (Figura 2). El Nevado de Chañi posee caminos de 1,2 $\mathrm{m}$ en la primera parte y $0,80 \mathrm{~m}$ en el último tramo hasta la cima (Vitry 2007); en el Volcán Llullaillaco el ancho oscila entre 1,5 a 2 metros y se reduce a poco menos de un metro en las pendientes fuertes (Vitry 2009); también se observó la misma característica en el Cerro Peladeros en Chile (Ibacache y Cantarutti 2006) y en el Cerro El Potro, donde Moyano (2009) observó un camino con un ancho variable entre $0,70 \mathrm{~m}$ y un metro. 
Tabla 1. Listado de montañas con evidencias de caminos ceremoniales. Indicadores A: camino; B: maderas en ladera; C: estructuras entre base y cima; D: ofrenda humana (capacocha).

List of mountains with evidence of ceremonial roads. Indicators A: Path; B: Hillside woods; C: Structures between base and top; D: Human offering (capacocha).

\begin{tabular}{|c|c|c|c|c|c|c|c|}
\hline \multirow{2}{*}{$\mathrm{N}^{\circ}$} & \multirow{2}{*}{ Nombre de montaña } & \multirow{2}{*}{$\underset{\mathrm{m}}{\text { Altura }}$} & \multirow{2}{*}{ Región / País } & \multicolumn{4}{|c|}{ Indicadores } \\
\hline & & & & A & B & $\mathrm{C}$ & D \\
\hline 1 & Acay & 5.716 & Salta, Argentina & $\mathrm{X}$ & & $\mathrm{x}$ & \\
\hline 2 & Amarillo & 3.600 & Jujuy, Argentina & $\mathrm{X}$ & & $\mathrm{X}$ & \\
\hline 3 & Ampato & 6.310 & Arequipa, Perú & $\mathrm{X}$ & $\mathrm{X}$ & $\mathrm{X}$ & $\mathrm{x}$ \\
\hline 4 & Bayo de Nazareno & 3.500 & Salta, Argentina & $\mathrm{X}$ & & $\mathrm{x}$ & \\
\hline 5 & Cachi, La Hoyada & 6.020 & Salta, Argentina & $\mathrm{X}$ & $\mathrm{X}$ & $\mathrm{X}$ & \\
\hline 6 & Cariquima o Wanapa & 5.365 & Iquique, Chile & $\mathrm{X}$ & & $\mathrm{x}$ & \\
\hline 7 & Colorado & 5742 & S.P. Atacama, Chile & & $\mathrm{x}$ & & \\
\hline 8 & Coropuna & 6.415 & Arequipa, Perú & $\mathrm{X}$ & & $\mathrm{x}$ & \\
\hline 9 & Las Cuevas & 4960 & Tucumán, Argentina & $\mathrm{X}$ & & $\mathrm{x}$ & \\
\hline 10 & Curiquinca & 5.769 & S.P. Atacama, Chile & $\mathrm{X}$ & & & \\
\hline 11 & Chachani & 6.057 & Arequipa, Perú & & $\mathrm{X}$ & $\mathrm{X}$ & $\mathrm{x}$ \\
\hline 12 & Chañi & 5.896 & Jujuy, Argentina & $\mathrm{X}$ & $\mathrm{X}$ & $\mathrm{x}$ & $\mathrm{X}$ \\
\hline 13 & Chiliques & 5.778 & S.P. Atacama, Chile & $\mathrm{X}$ & $\mathrm{x}$ & $\mathrm{x}$ & \\
\hline 14 & Cerro Doña Ana & 5.690 & Coquimbo, Chile & & $\mathrm{x}$ & $\mathrm{X}$ & \\
\hline 15 & Las Flechas & 5.350 & San Juan, Argentina & $\mathrm{X}$ & $\mathrm{X}$ & & \\
\hline 16 & Fundición de Nazareno & 5.050 & Salta, Argentina & $\mathrm{X}$ & & $\mathrm{x}$ & \\
\hline 17 & Guana Guane & 5.050 & Atacama, Chile & & $\mathrm{X}$ & $\mathrm{x}$ & \\
\hline 18 & Hanac Pacha & 5.920 & Arequipa, Perú & & $\mathrm{X}$ & & \\
\hline 19 & Incahuasi & 6.638 & Catamarca, Argentina & $\mathrm{X}$ & $\mathrm{X}$ & $\mathrm{X}$ & \\
\hline 20 & Isluga & 5.530 & Atacama, Chile & & $\mathrm{X}$ & $\mathrm{X}$ & \\
\hline 21 & Juriques & 5.662 & S.P. Atacama, Chile & & $\mathrm{X}$ & $\mathrm{x}$ & \\
\hline 22 & Licancabur & 5.921 & Atacama, Chile & $\mathrm{X}$ & $\mathrm{X}$ & $\mathrm{x}$ & \\
\hline 23 & Llampos & 1.557 & Copiapó, Chile & $\mathrm{X}$ & & $\mathrm{X}$ & \\
\hline 24 & Llullaillaco & 6.739 & Salta, Argentina & $\mathrm{X}$ & $\mathrm{X}$ & $\mathrm{X}$ & $\mathrm{x}$ \\
\hline 25 & Mercedario & 6.770 & San Juan, Argentina & $\mathrm{x}$ & $\mathrm{X}$ & $\mathrm{x}$ & \\
\hline 26 & Miño & 5.600 & Atacama, Chile & $\mathrm{x}$ & & $\mathrm{x}$ & \\
\hline 27 & Los Mogotes & 5.380 & San Juan, Argentina & $\mathrm{x}$ & & & \\
\hline 28 & Negro Overo & 6.050 & La Rioja, Argentina & & $\mathrm{X}$ & $\mathrm{x}$ & \\
\hline 29 & Las Palas & 4.993 & Huasco, Chile & & $\mathrm{X}$ & $\mathrm{x}$ & \\
\hline 30 & Paniri & 5.946 & Atacama, Chile & & $\mathrm{X}$ & $\mathrm{x}$ & \\
\hline 31 & Pariacaca & 5.730 & Yauyos, Perú & & $\mathrm{x}$ & $\mathrm{x}$ & \\
\hline 32 & Pichu Pichu & 5.634 & Arequipa, Perú & $\mathrm{X}$ & $\mathrm{X}$ & $\mathrm{x}$ & $\mathrm{x}$ \\
\hline 33 & Pili & 6.060 & S.P. Atacama, Chile & $\mathrm{x}$ & & $\mathrm{x}$ & \\
\hline 34 & El Plomo & 5.425 & Santiago, Chile & $\mathrm{X}$ & $\mathrm{x}$ & $\mathrm{x}$ & $\mathrm{x}$ \\
\hline 35 & El Potro & 5.830 & San Juan, Argentina & $\mathrm{X}$ & & $\mathrm{x}$ & \\
\hline 36 & Pular & 6.225 & S.P. Atacama, Chile & & $\mathrm{X}$ & $\mathrm{X}$ & \\
\hline 37 & Los Puntiudos & 2.000 & La Serena, Chile & & $\mathrm{x}$ & $\mathrm{x}$ & \\
\hline 38 & Quewar & 6.130 & Salta, Argentina & $\mathrm{X}$ & & $\mathrm{x}$ & $\mathrm{x}$ \\
\hline 39 & Quimal & 4.300 & S.P. Atacama, Chile & & $\mathrm{X}$ & $\mathrm{X}$ & \\
\hline 40 & Sairecabur & 5.970 & Atacama, Chile. & & $\mathrm{x}$ & $\mathrm{X}$ & \\
\hline 41 & Salla & 5.036 & Atacama, Chile & $\mathrm{x}$ & & & \\
\hline 42 & Sara Sara & 5.505 & Arequipa, Perú & & $\mathrm{X}$ & $\mathrm{x}$ & $\mathrm{X}$ \\
\hline 43 & Siete Hermanas Maricunga & 4.878 & Copiapó, Chile & $\mathrm{X}$ & & $\mathrm{x}$ & \\
\hline 44 & Socompa & 6.031 & Salta, Argentina & $\mathrm{X}$ & $\mathrm{X}$ & $\mathrm{x}$ & \\
\hline 45 & Tambillos & 5.747 & San Juan, Argentina & $\mathrm{x}$ & $\mathrm{X}$ & $\mathrm{x}$ & \\
\hline 46 & Tata Jachura & 5.252 & Iquique, Chile & $\mathrm{X}$ & & $\mathrm{x}$ & \\
\hline 47 & El Toro & 6.380 & San Juan, Argentina & $\mathrm{X}$ & & $\mathrm{x}$ & $\mathrm{x}$ \\
\hline 48 & Tórtolas & 6.323 & San Juan, Argentina & $\mathrm{x}$ & $\mathrm{X}$ & $\mathrm{x}$ & \\
\hline 49 & Yeso & 4.624 & Ancash, Perú & $\mathrm{X}$ & & & \\
\hline & & & & 33 & 30 & 42 & 9 \\
\hline
\end{tabular}




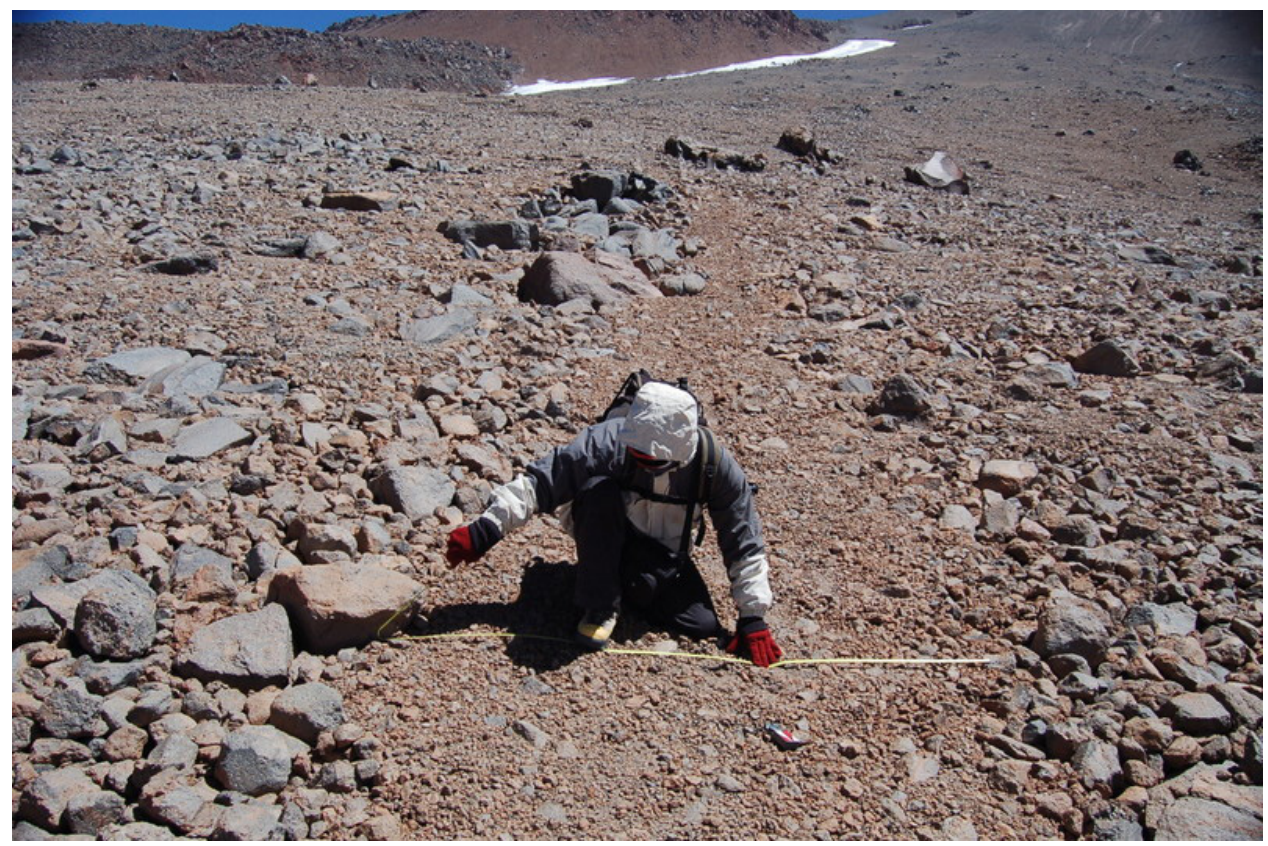

Figura 2. Camino ceremonial del volcán Llullaillaco, se aprecia el ancho constante.

Ceremonial route of the Llullaillaco volcano, the constant width can be seen.

\section{Presencia de muros de contención}

Los muros de contención, por su alta visibilidad, son los que dieron las primeras señales de la existencia de caminos en las montañas. Los mismos, pese a las dificultades de trabajar a tanta altura, poseen idénticos rasgos arquitectónicos que los de las partes más bajas, es decir, selección de rocas con caras planas, las cuales son dispuestas formando un muro parejo, sin protuberancias o salientes que denoten irregularidades, como los observados en la ladera oriental del Nevado de Cachi (Figura 3).

\section{Trazados en zigzag y/o geomórficos}

Otro indicador relacionado con estos caminos formalmente construidos y que se puede observar en muchas montañas (Cachi, Chañi, Llullaillaco, Licancabur, Pichu Pichu, Acay, etc.) es el trazado en zigzag siguiendo líneas rectas y quebrando en ángulos agudos. Se observaron también algunos casos de caminos que no responden a un trazado zigzagueante, sino que se acomodan al relieve por donde discurren, son geomórficos (Berenguer et al. 2005:14), asentándose siempre sobre las partes más firmes y laderas más suaves de los cerros, ejemplos de éstos hallamos en el Llullaillaco, Quewar y Socompa (Figura 4).

\section{Ubicación en laderas orientales}

Se trata de una regularidad observada en la mayoría de las montañas que poseen restos arqueológicos y caminos, sin embargo existen excepciones registradas en algunas montañas de Chile (Ibacache y Cantarutti 2006; Ibacache et al. 2016) y Bolivia (Cruz et al. 2013) donde los caminos se encuentran marcadamente en el occidente. Nonecesariamente se trata de que se localicen en el E propiamente tal, sino en todo el espectro que abarca desde el N hasta el SSE. Suponemos que, tanto los sitios como de los caminos, están emplazados en estas laderas para aprovechar las primeras luces y calor del sol naciente y la necesidad de poder calentarse con los primeros rayos en lugares tan fríos como los montañosos.

\section{Infraestructura asociada}

Como se aprecia en la Tabla 1 la altitud de las montañas con evidencias arqueológicas llega a superar los $6.700 \mathrm{~m}$ y promedian en $5.500 \mathrm{~m}$, lo cual genera una necesidad vital de poder ir ascendiendo paulatinamente hasta la cima. Por otra parte, las condiciones ambientales son muy cambiantes y en cuestión de pocas horas se puede pasar de un día soleado a una tormenta de nieve, de viento o eléctrica. Se ha registrado en la mayor parte de las montañas la presencia de pequeños recintos a diferentes alturas. Los mismos son de paredes bajas (la mayoría no supera los 1,5 m), puertas de acceso bajas y estrechas -para poderlas cerrar con pocos bultos- y conservar el calor, techos construidos con tirantes de madera que a veces superan $\operatorname{los} 3 \mathrm{~m}$ de largo, paja, cuerdas de fibra vegetal y rocas planas en 


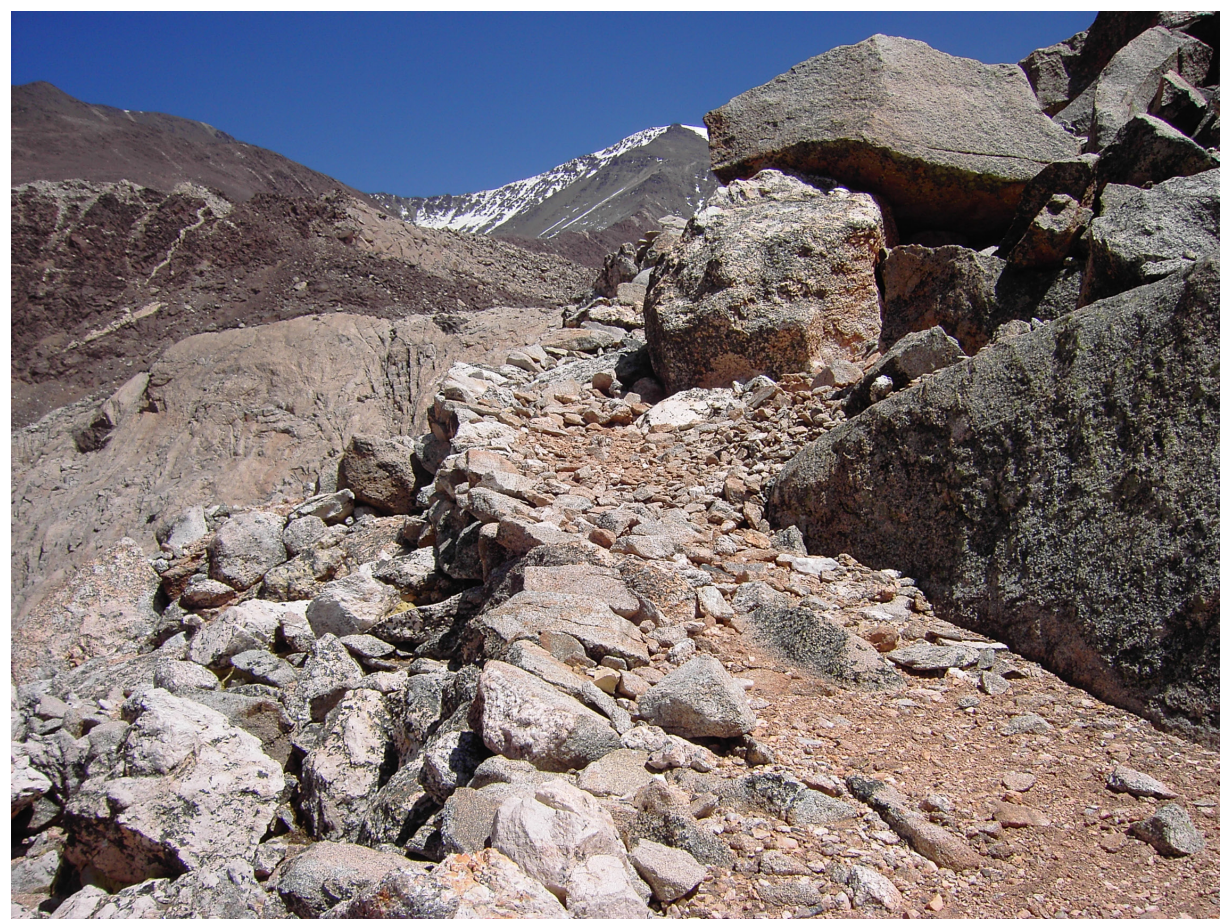

Figura 3. Muros de contención de un segmento de camino en el Nevado de Cachi.

Retaining walls of a road section in Nevado de Cachi.

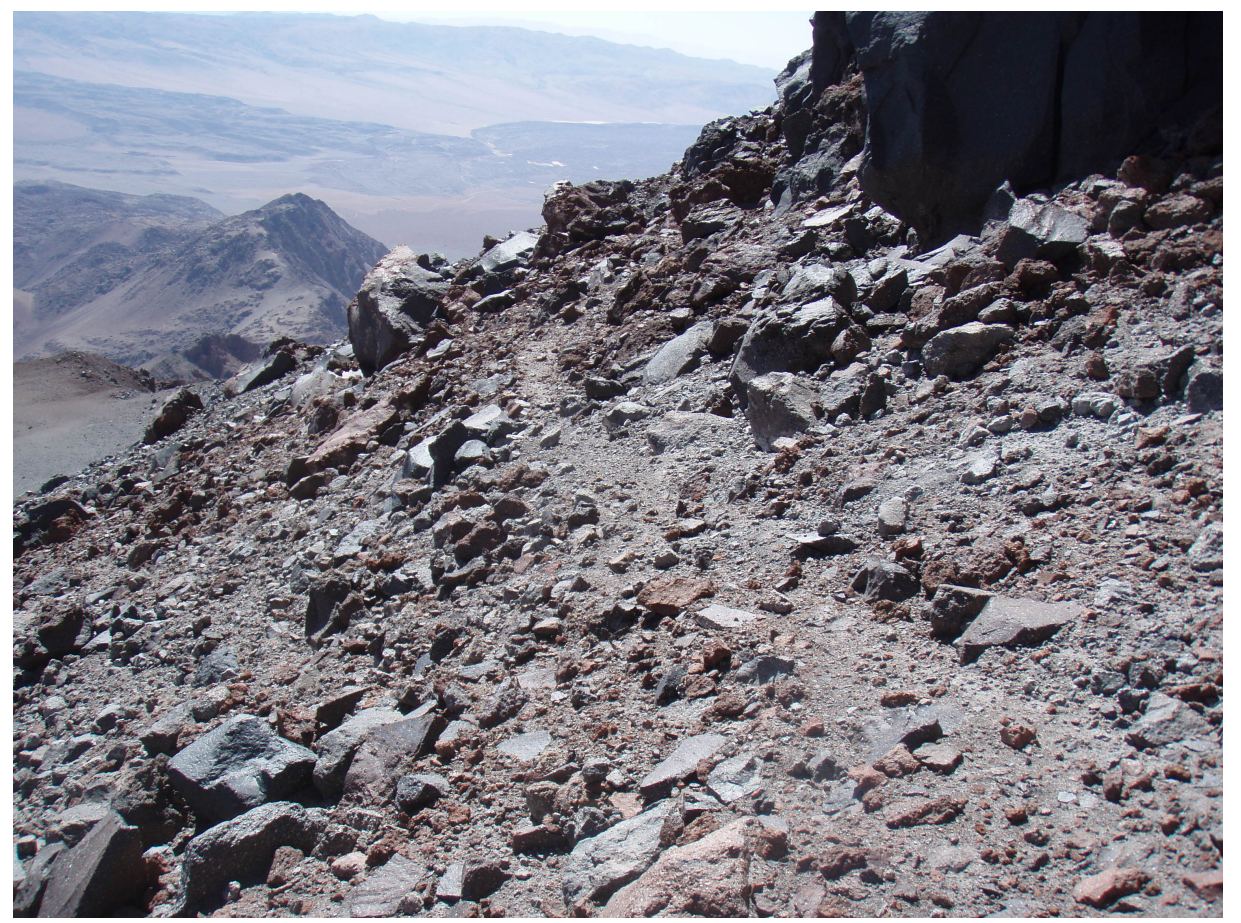

Figura 4. Segmento de camino geomórfico en el volcán Socompa.

Geomorphic road section in the Socompa volcano. 
la parte superior (Figura 5). No obstante, existen también diferencias en las técnicas y calidades constructivas y la energía invertida en ellas, tal como se puede apreciar en el Volcán Llullaillaco (Vitry 2009).

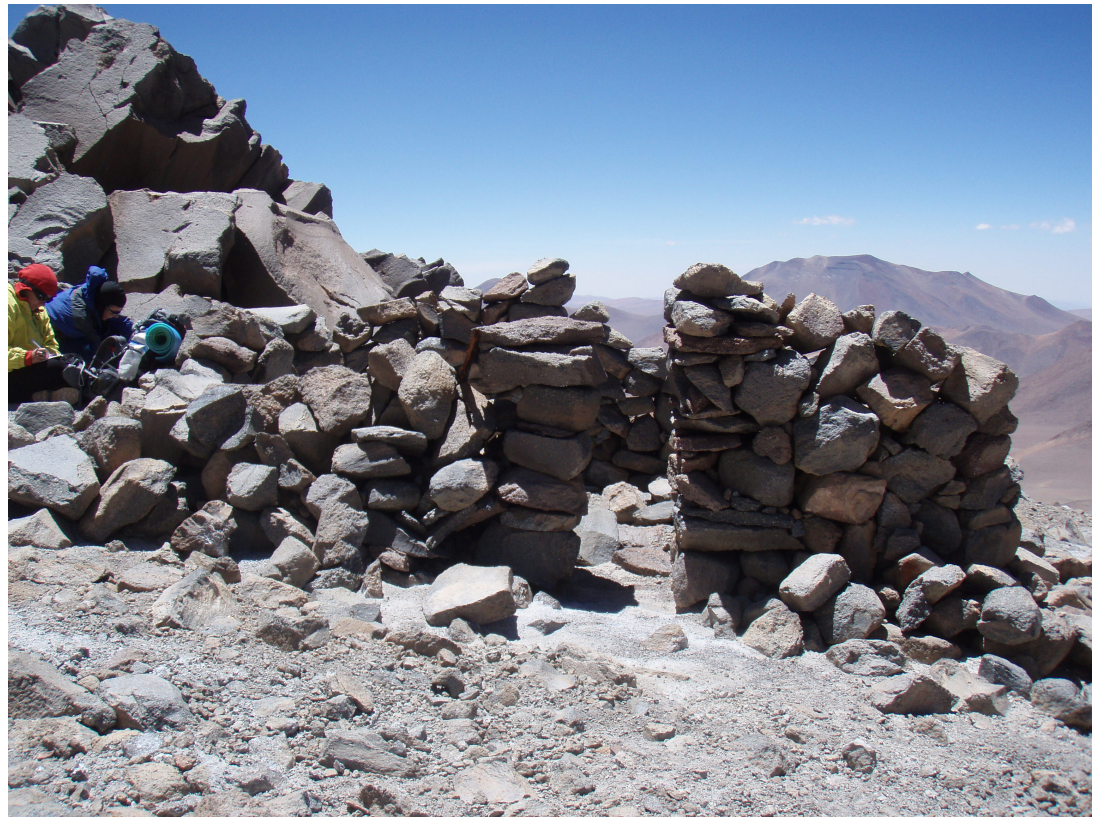

Figura 5. Infraestructura asociada al camino ceremonial del volcán Llullaillaco a $5.650 \mathrm{msm}$.

Infrastructure associated with the ceremonial path of the Llullaillaco volcano at 5,650 asl.

\section{Presencia de marcadores espaciales}

Se registrólapresencia de mojones, apachetas y sayhuas tanto en cimas como en laderas, pasos montañosos y bases de las montañas (Vitry 2002). Los mojones suelen estar relacionados con los caminos, ya que lo van jalonando de trecho en trecho (no en todos los casos, pero es muy frecuente). Se observaron mojones de gran porte y alta visibilidad en laderas de montañas, como el caso del Volcán Aracar y Nevado de Cachi (Salta, Argentina), que estarían indicando caminos (Figura 6). Por otra parte, además de lo antedicho, Cruz indica la presencia de sayhuas y da a conocer por primera vez rocas con figuras serpentiformes hechas por termo-alteración que van jalonando e indicando la vía de acceso a los adoratorios de altura en el altiplano boliviano (Cruz 2015; Cruz et al. 2013). En la Sierra de Famatina (La Rioja), que es considerada como una huaca de alcance interregional, Martín $(2015,2017)$ observa una serie de marcadores espaciales (plataformas, mojones y wankas) en un camino ceremonial que se dirige a la montaña sagrada.

\section{Maderas o amojonamientos en curvas y cambios de dirección del camino}

Esta regularidad la observamos en la mayoría de las montañas del Norte argentino (Vitry 2006, 2007,
2009, 2017), y de hecho quizás sea uno de los principales indicadores de caminos, pues en los zigzagueantes se colocaba en cada curva aguda una madera, de manera tal que, ante cualquier nevada que tapara el camino (hecho muy frecuente en la montaña), el mismo se definía por los pequeños postes que sobresalían y unían los rectilíneos derroteros. El registro arqueológico da sobradas muestras de estos troncos del ancho de un brazo y longitudes que oscilan entre los $0,50 \mathrm{~m}$ y $1,5 \mathrm{~m}$ (Figura 7). En este punto debemos considerar también como un indicador arqueológico de camino las interesantes observaciones realizadas en numerosas montañas de Potosí (Bolivia), donde existen rocas con grabados serpentiformes realizados por termo-alteración, las cuales están directamente asociadas a las vías de acceso a los adoratorios de altura, uniendo sitios de base, ladera y cumbre (Cruz 2015).

9. Presencia de contextos de ofrendas vinculados con arquitectura y objetos incas, en algunos casos también capacochas

Todo parece indicar que la construcción de caminos ceremoniales está relacionada directamente con la importancia de la montaña, la cual se puede apreciar en la cantidad de energía invertida en ella a través de la 


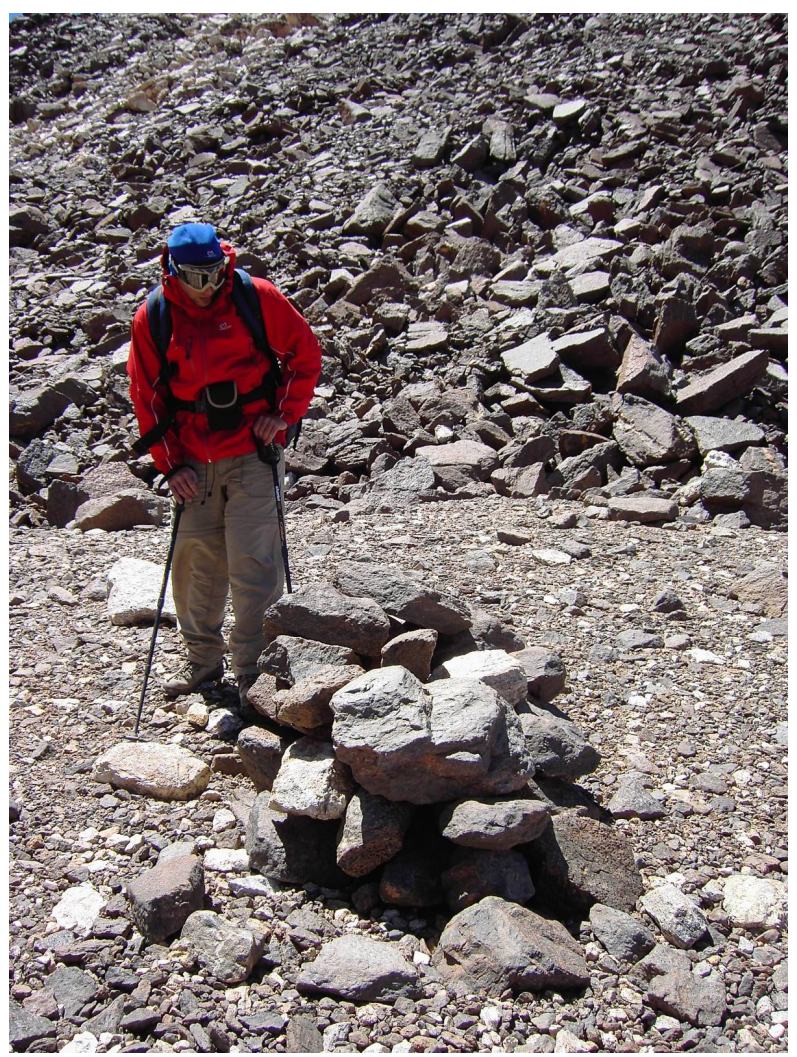

Figura 6. Mojón ubicado sobre la ladera del Nevado de Cachi, antes de un cambio de pendiente.

Cairn located on the slope of the Nevado de Cachi before a slope change.

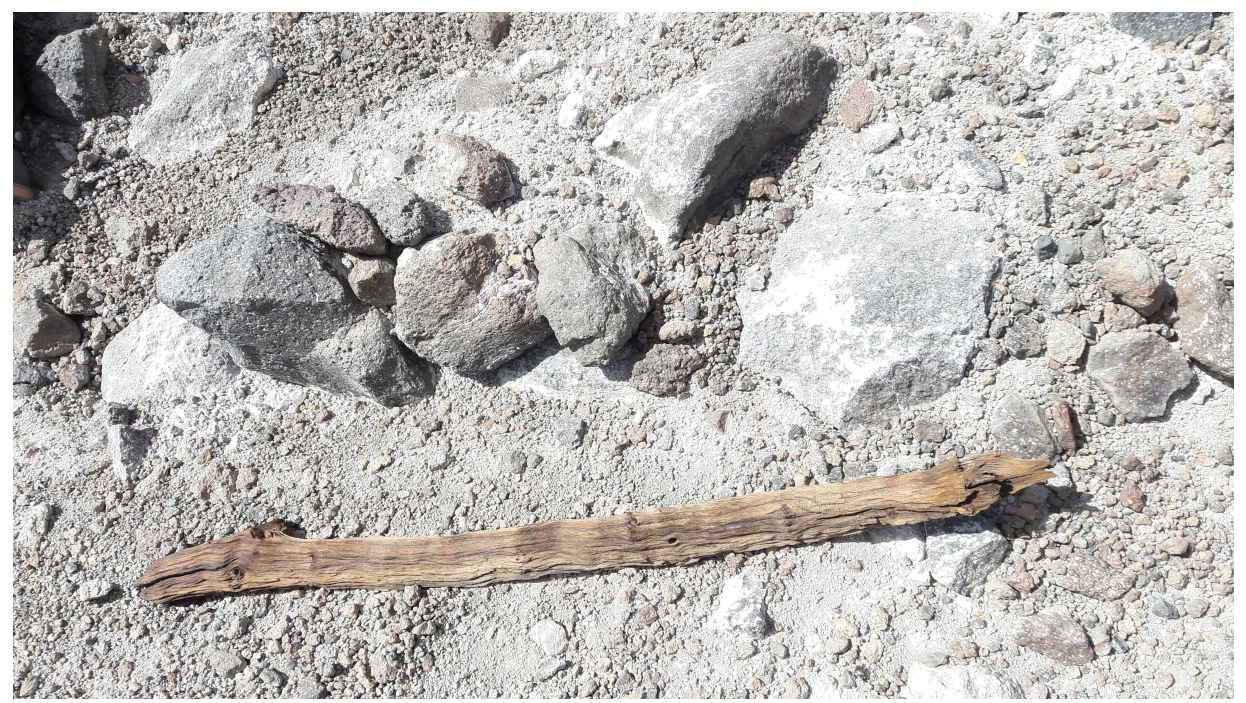

Figura 7. Madera demarcatoria de curvas y segmento de camino con muro lateral en el volcán Llullaillaco.

Demarcatory wood of curves and road section with lateral wall in the Llullaillaco volcano. 
construcción de sitios intermedios, caminos con muros de contención, maderas traídas de centenares de kilómetros de distancia y la ofrenda mayor consistente en individuos enterrados (Reinhard 1993-1994; Reinhard y Ceruti 2000). Uno de los casos más significativos y que mayor cantidad de infraestructura posee es el Volcán Llullaillaco, donde además de las estructuras ubicadas a diferentes alturas y hasta la cima, tiene un cementerio en su base, donde se identificaron numerosos entierros que posiblemente pertenecieron a los mitimaes de los incas que trabajaron en la construcción del adoratorio (Mignone 2010). En toda la cordillera se identificaron 15 montañas con ofrendas humanas, de las cuales se extrajeron 27 cuerpos (Vitry 2008); sin embargo, hay muchas montañas que poseen una gran inversión constructiva pero sin que se hayan encontrado ofrendas humanas, como es el caso del Volcán Licancabur Figura 8 (Reinhard 1983).

\section{Plataformas en antecumbres}

Fueron definidas por Raffino (1981) como plataformas artificiales y por Beorchia Nigris (1985) como explazos ceremoniales. Suelen ser recintos rectangulares y excepcionalmente circulares, casi al nivel del suelo, con una o dos hiladas de rocas, en algunos casos están orientados según los puntos cardinales o bien indicando algún geosímbolo o lugares distantes, señalando otras montañas o huacas. Estas plataformas se localizan en la mayoría de los casos por debajo de la cima (Tabla 2) y su distancia y diferencia altitudinal puede ser de pocos metros respecto a la cumbre, como el caso del Llullaillaco, Quewar, Plomo, etc., o bien a bastante distancia de ella como en el Nevado de Chuscha y el Aconcagua (Schobinger 1986, 1998, 2008). En la Tabla 2 solo se presentan las plataformas donde se localizaron enterratorios humanos en contextos de capacochas.
Tabla 2. Ubicación altitudinal de las plataformas ceremoniales respecto a la cumbre de las montañas.

Altitudinal location of the ceremonial platforms with respect to the summit of the mountains.

\begin{tabular}{cccc}
\hline Montaña & $\begin{array}{c}\text { Altura de la } \\
\text { Montaña } \\
(\mathrm{m})\end{array}$ & $\begin{array}{c}\text { Altura de la } \\
\text { plataforma } \\
(\mathrm{m})\end{array}$ & Ubicación \\
\hline Aconcagua & 6.959 & 5.250 & Mendoza - Argentina \\
\hline Ampato & 6.310 & $5.800-6.300$ & Arequipa - Perú \\
\hline Chachani & 6.057 & 5.851 & Arequipa - Perú \\
\hline Chañi & 5.896 & 5.880 & Jujuy - Argentina \\
\hline Chuscha & 5.420 & 5.120 & Salta - Argentina \\
\hline Coropuna & 6.415 & 5.000 & Arequipa - Perú \\
\hline El Plomo & 5.425 & 5.400 & Santiago - Chile \\
\hline El Toro & 6.380 & 6.300 & San Juan - Argentina \\
\hline Llullaillaco & 6.739 & 6.720 & Salta - Argentina \\
\hline Misti & 5.850 & 5.822 & Arequipa - Perú \\
\hline Pichu Pichu & 5.634 & 5.630 & Arequipa - Perú \\
\hline Quewar & 6.130 & 6.100 & Salta - Argentina \\
\hline Sara Sara & 5.505 & 4.565 & Arequipa - Perú \\
\hline
\end{tabular}

11. Relación visual de los edificios que conforman la infraestructura vial respecto de la cima más importante de la montaña

Una observación que realizamos y que hasta el momento es recurrente, es que cada uno de los sitios ubicados entre la base y cima de la montaña posee una conexión visual con la cumbre o antecumbre donde se localiza la plataforma ceremonial.

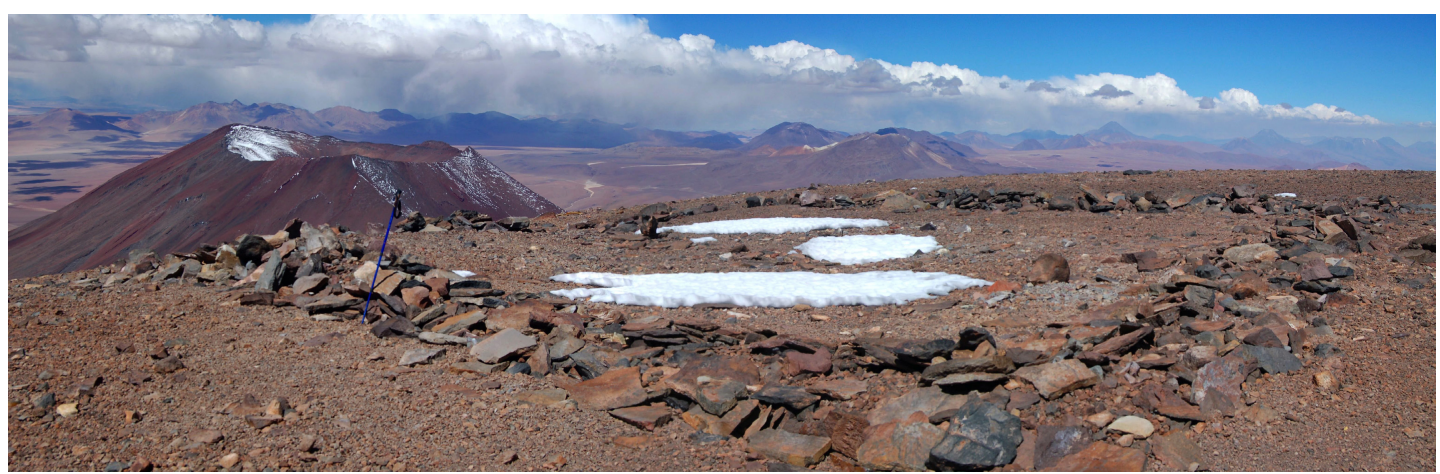

Figura 8. Plataforma ceremonial en la cima del Volcán Licancabur.

Ceremonial platform on the top of the Licancabur volcano. 
12. Cumbres con poca o nula construcción de infraestructura

Por lo general, en la cúspide de las montañas no suele haber mucha infraestructura, las mismas se localizan inmediatamente por debajo de la parte más alta (Tabla 2). Esta forma de construir el espacio ceremonial en las montañas, con una diferenciación entre una parte alta y otra baja, es frecuente en el patrón de asentamiento e ideología incaica. En otro trabajo hipotetizamos sobre la posible relación cumbre - ushnu (Vitry 2008:57), donde se propone que el ushnu estaría representado por la cima de la montaña, convirtiéndose en el punto de mayor sacralidad del paisaje, el lugar de contacto entre lo terrenal y celestial o vínculo con el hanan pacha.

\section{Discusión y Palabras Finales}

Las preguntas iniciales que nos realizamos, fueron respondidas en parte mediante el planteamiento de los indicadores arqueológicos y el registro de la muestra de montañas analizadas, sin embargo, es necesario profundizar y discutir sobre algunos temas.

Desde el punto de vista constructivo podemos concluir que los caminos ceremoniales no se diferencian de cualquier otra vialidad incaica, pues entran dentro de las categorías y características ya definidas por muchos autores (Berenguer et al. 2005; González 2017; Hyslop 1984, 1992; Martín 2005; Raffino 1981; Vitry 2000,
2005; entre otros). De hecho, se observan en el registro arqueológico caminos despejados, despejados y amojonados, con muros laterales y con rampa artificial; respecto al trazado se presentan los típicos zigzag, las líneas rectas y también los anchos constantes. En el caso de los caminos geomórficos que mencionamos más arriba, si bien no siguen los trazados convencionales (zigzag y rectilíneo) conservan las demás características constructivas propias de las vialidades incaicas, resultando ser dicha construcción una respuesta adaptativa al ambiente. Siendo el camino de similares características que el resto de las vialidades incas, solo resta decir que lo que le otorga el carácter de ceremonial o ritual es el adoratorio, el $a p u$, el espacio sagrado por donde transita (Bauer 2000; Elíade 1994).

Con relación a la infraestructura asociada a estos sistemas viales de montaña y, a diferencia de los construidos en ambientes no tan extremos, donde existe una secuencia de sitios de enlace (Nielsen et al. 2006) que guardan cierta equidistancia, podemos decir que en estos caminos de montaña la equidistancia es altitudinal y en menor medida longitudinal, pues en este tipo de ambientes ni los chasquis pueden correr ni las llamas ascender. Por ejemplo, en el Volcán Llullaillaco existen siete sitios de enlace en relación al camino, estos son: Filo Norte 5.000 m, Tambo 5.290 m, Laderas Bajas 5.580 m, Cota de Agua $5.800 \mathrm{~m}$, Laderas Altas $6.300 \mathrm{~m}$, Portezuelo $6.500 \mathrm{~m}$ y Adoratorio de la Cumbre 6.720 m, hallándose la cima a $6.739 \mathrm{~m}$ (Figura 9). Si observamos la diferencia altitudinal

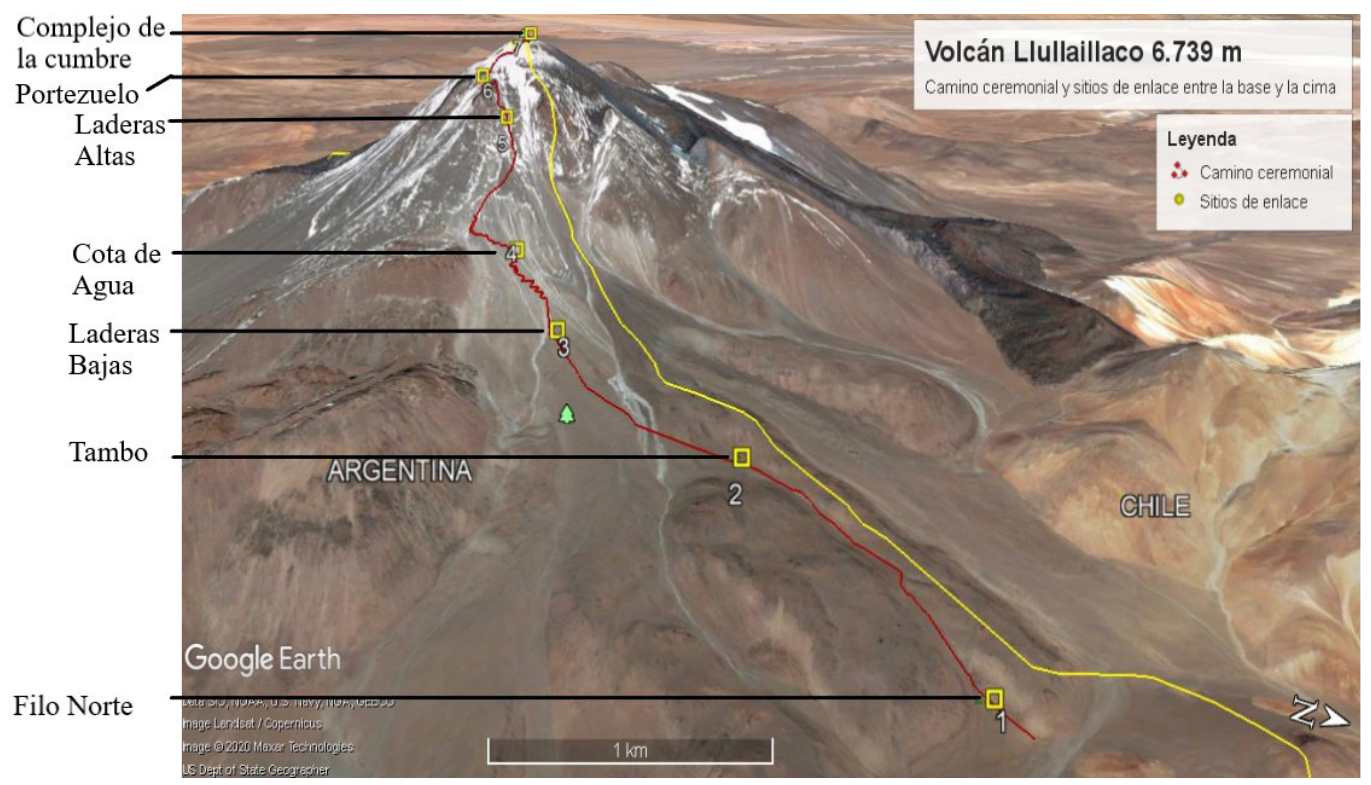

Figura 9. Distribución de los sitios de enlace en relación al camino ceremonial en el Volcán Llullaillaco. (1) Filo Norte 5.000 m, (2) Tambo 5.290 m, (3) Laderas Bajas 5.580 m, (4) Cota de agua 5.800 msm, (5) Laderas Altas 6.300 m, (6) Portezuelo 6.500 m y (7) Adoratorio de la cumbre $6.720 \mathrm{~m}$.

Distribution of the link sites in relation to the ceremonial path at the Llullaillaco volcano. (1) Filo Norte 5,000 m, (2) Tambo 5,290 m, (3) Laderas Bajas 5,580 m, (4) Cota de agua 5,800 mals, (5) Laderas Altas 6,300 m, (6) Portezuelo 6,500 m, and (7) Adoratorio de la cumbre $6,720 \mathrm{~m}$. 
entre cada uno de los sitios a lo largo de los 7,23 km de recorrido desde la base a la cima, donde salva un desnivel de $1.739 \mathrm{~m}$, notaremos que la altura promedio que separa a cada sitio es de aproximadamente $300 \mathrm{~m}$ y la distancia 1,12 km (Tabla 3). Esta equidistancia vertical y longitudinal tiene total sentido en el tipo de terreno, además de las condiciones ambientales y generales de la época; en la práctica del montañismo actual la equidistancia vertical de ascenso oscila entre 400 y 600 metros diarios para lograr una óptima aclimatación del cuerpo a la altitud cada vez mayor, huelga decir que hoy se dispone de un equipo sofisticado que es bastante liviano y abrigado. Se debe tener en cuenta que las condiciones de equipamiento en épocas prehispánicas eran muy precarias, empezando por el calzado, que consistía en unos zapatones de lana de varias capas (Vitry 2014), los cuales seguramente no resistirían a las fuertes tempestades que a veces se manifiestan en las montañas. Suponemos que la estrategia consistió en realizar numerosos "campamentos" o sitios de enlace a relativamente poca distancia, con la finalidad de poder guarecerse de las imprevistas y repentinas inclemencias climáticas. Existen varios ejemplos de adoratorios de altura con varios asentamientos de enlace, como el Licancabur, Quewar, Aconcagua, Cachi, Socompa, Chañi y otros (Beorchia Nigris 1985; Schobinger 1998; Vitry 1997).

Ya nos referimos en otro artículo sobre la vinculación de las montañas con el Qhapaq Ñan y de su importancia intrínseca (Vitry 2017) y, en consonancia con esta línea de trabajo, tenemos las investigaciones realizadas en la Sierra de Famatina, donde en relación directa con el camino se localizaron una serie de marcadores espaciales de carácter ceremonial, generando un estrecho vínculo entre la montaña sagrada y la vialidad (Martín 2015, 2017); asimismo se destacó el trabajo de Besom (2009) quien realiza un exhaustivo y prolijo análisis de los cronistas que se refirieron al culto de las montañas

Tabla 3. Relación del camino con los sitios de enlace del Volcán Llullaillaco de la base a la cumbre, tanto en distancia como en altura.

Relationship of the road with the link sites of the Llullaillaco volcano from the base to the summit, both in distance and height wise.

\begin{tabular}{lcccc}
\hline Sitio de enlace & $\begin{array}{c}\text { Altitud } \\
(\mathrm{msm})\end{array}$ & $\begin{array}{c}\text { Diferencia } \\
\text { de altura } \\
(\mathrm{m})\end{array}$ & $\begin{array}{c}\text { Distancia } \\
\text { Relativa } \\
(\mathrm{km})\end{array}$ & $\begin{array}{c}\text { Distancia } \\
\text { Absoluta } \\
(\mathrm{km})\end{array}$ \\
\hline 1. Filo Norte & 5.000 & - & - & \\
2. Tambo & 5.290 & 290 & 1,74 & 1,74 \\
3. Laderas Bajas & 5.580 & 290 & 1,32 & 3,46 \\
4. Cota de Agua & 5.800 & 210 & 1,10 & 4,56 \\
5. Laderas Altas & 6.300 & 500 & 1,5 & 6,06 \\
6. Portezuelo & 6.500 & 200 & 0,5 & 6,56 \\
7. Adoratorio de & 6.720 & 220 & 0,6 & 7,16 \\
la Cumbre & 6.739 & 19 & 0,07 & 7,23 \\
8. Cumbre & & & & \\
\hline
\end{tabular}

clasificándolos en quince categorías, un trabajo para tener en cuenta ante la pregunta sobre la sacralidad de las montañas.

Podemos proponer que las montañas tienen una doble función, ya que como grandes hitos marcan un punto de referencia en la organización espacial; asimismo, como geosímbolos le otorgan una historia al paisaje que contribuye a la consolidación de esa cartografía oral andina (Sanhueza 2012) que actúa como pivote de la identidad y de la legitimación de un nuevo orden socioespacial propuesto por los incas en su proceso expansión.

Porotra parte, no debemos olvidar laimportanciaque tiene la imponencia visual de la montaña en esa ruptura de la horizontalidad y su proyección vertical hacia el cielo, hecho que llevó a numerosas religiones del mundo a convertirlas en objetos de culto y axis mundi (Eliade 1994); asimismo, su belleza, su íntima relación con la minería (Cruz 2009; Salazar et al. 2013), como también su humanización y vínculo directo con las sociedades hasta tiempos modernos, hecho que ha demostrado Bastien (1978) en la denominada Montaña del Cóndor, en cuyos diferentes pisos ecológicos las comunidades Kallawayas de Bolivia basan su organización social y espacial.

En los últimos años, a raíz del proceso de inclusión del Qhapaq Ñan en la Lista del Patrimonio Mundial de UNESCO, se ha avanzado mucho en el registro cartográfico, sin embargo, queda pendiente un trabajo más integral para poder vincular las redes viales regionales y sitios asociados con los adoratorios de altura y el Qhapaq Ñan.

Nuestros estudios revelaron la existencia de 226 montañas que fueron utilizadas con fines rituales por los Incas, sobre ese total y hasta el momento, registramos 58 de ellas con evidencias directas o indirectas de caminos ceremoniales entre la base y la cima, lo cual representa el $25,66 \%$ sobre el total. Respecto a capacochas, solamente en 15 montañas se localizaron ofrendas humanas, esto es el $6,6 \%$.

Este primer intento de sistematización de los caminos ceremoniales del Tawantinsuyu, basada en determinados indicadores arqueológicos, nos permite dimensionar la cantidad de energía invertida en la construcción de vías e infraestructura asociada para la apropiación ritual de estos espacios tan particulares, cuya sacralidad, posiblemente, fue creciendo con el tiempo, con las prácticas rituales y las ofrendas entregadas al apu.

Queda claro que cuando los incas ascendían a las montañas para realizar ofrendas no siempre se trataba de un ascenso expeditivo, sino más bien de todo un proceso previo de estudio de la morfología de la montaña, construcción de caminos y estructuras asociadas, transporte de madera, paja, vasijas y otros elementos desde centenares de kilómetros. Esto en el llano puede figurarse como algo cotidiano y sin mayor trascendencia, pues hemos asumido a través del caravaneo las largas distancias recorridas en 
el ámbito andino desde hace milenios, sin embargo, no quisiera dejar de destacar la dimensión humana de lo que debió haber implicado trabajar por arriba de los 5.000 e incluso $6.700 \mathrm{~m}$ de altura. Obviamente no se trató de personas súper dotadas, sino de humanos como cualquiera de nosotros, quizás un poco más acostumbrados a los rigores del frío cordillerano, pero con un equipo de abrigo bastante precario en comparación con los actuales. Las montañas poseen un ciclo natural respecto al estado del tiempo, y para poder transitar por ellas sin mayores riesgos de ser sorprendidos por una tormenta de cualquier tipo hay que conocerlas. Si bien las fuentes no hacen mención a algún personaje especializado o "camayoc" en estas actividades. y conociendo el nivel organizativo de los incas y los requerimientos logísticos de un ascenso a las altas montañas, no caben dudas que debieron haber existido especialistas en montañas, personas que sabían perfectamente cómo y cuándo moverse en ellas, donde ubicar los "refugios" y trazar los caminos, expertos planificadores de actividades para optimizar los tiempos de estadía en estos lugares extremos donde cualquier equivocación podía costarles la vida.

Los caminos ceremoniales debieron tener tanta sacralidad como el resto de los lugares en los adoratorios de altura, transitar por ellos hacía posible el acercamiento a la máxima deidad incaica, el Sol. Esto lo pudimos apreciar en los calzados que los incas emplearon para ascender el Volcán Llullaillaco, los cuales estaban equipados con una serie de elementos de carácter simbólico relacionados con la protección espiritual de quien los portaba, para luego de haber pisado los lugares sagrados cerca del cielo, ofrendar los calzados en una roca-huaca en la base de la montaña (Vitry 2014).

A través de las materialidades no podemos "ver" la sacralidad, pero si dimensionar la importancia que estos caminos y montañas tuvieron durante el siglo XV y parte del XVI para los Incas y sus estrategias expansivas en tierras lejanas.

Agradecimientos: El Presente trabajo se realizó en el marco del Proyecto de Investigación Tipo "A" $\mathrm{N}^{\circ}$ 2108/0 y N 2523 bajo mi dirección, dependiente del Consejo de Investigaciones de la Universidad Nacional de Salta. Agradezco a los miembros del equipo, particularmente a Federico Viveros por la elaboración del mapa; a Diego Ashur, de la Subsecretaría de Patrimonio Cultural por brindarme el espacio y las oportunidades de realizar trabajos de campo; a Jorgelina Flores Barrantes por las traducciones, su acompañamiento y apoyo; al montañista chileno Juan Carlos Carmona Risi por los datos aportados y la confianza; a mi querido amigo y pionero de la Arqueología de Alta Montaña Antonio Berochia Nigris, por su amistad, afecto y por haber cedido su valioso archivo del CIADAM al Museo de Arqueología de Alta Montaña; a los evaluadores del presente trabajo que gracias a sus críticas constructivas reorientaron el manuscrito e hicieron posible su mejora.

\section{Referencias Citadas}

Astuhuamán Gonzales, C. 1999. El "Santuario de Pariacaca". Alma Mater 17:127-147.

Bauer, B. 2000. El Espacio Sagrado de los Inkas. El Sistema de Ceques del Cuzco. Centro de Estudios Regionales Andinos "Bartolomé de las Casas", Cuzco.

Beorchia Nigris, A. 1985. El Enigma de los Santuarios Indígenas de Alta Montaña. Universidad Nacional de San Juan, San Juan.

Beorchia Nigris, A. (compil.) 2001. Revista del Centro de Investigaciones Arqueológicas de Alta Montaña (CIADAM). Tomo 6ª años 1987-1999. Universidad Católica de La Plata, La Plata.

Berenguer R.,+J., I. Cáceres R., C. Sanhueza y P. Hernández V. 2005. El Qhapaqñan en el Alto Loa, norte de Chile: Un estudio micro y macromorfológico. Estudios Atacameños 29:7-39.

Besom, T. 2009. Of Summits and Sacrifice an Ethnohistoric Study of Inka Religious Practices. University of Texas Press, Austin.

Bonnemaison, J. 1992. Le territoire enchanté. Croyances et territorialités en Mélanésie. Géographie et Culture 3:71-88.

Castro, V., V. Varela, C. Aldunate y E. Araneda 2004. Principios orientadores y metodología para el estudio del Qhapaq Nan en Atacama: desde el portezuelo del Inka hasta Río Grande. Chungara Revista de Antropología Chilena 36 (2):439-451.
Ceruti, M.C. 2003. Llullaillaco. Sacrificios y ofrendas en un Santuario Inca de Alta Montaña. EUCASA., Universidad Católica de Salta, Salta.

Cruz, P. 2009. Huacas olvidadas y cerros santos. Apuntes metodológicos sobre la cartografía sagrada en los Andes del sur de Bolivia. Estudios Atacameños 38:55-74.

Cruz, P. 2015. Tatala Purita o el Influjo del Rayo, Arte Rupestre Anicónico en las Altas Tierras Surandinas (Potosí, Bolivia). Boletín SIARB 29:51-70.

Cruz, P., E. Crubézy y P. Gérard 2013. Los adoratorios de altura inkaicos. Una mirada desde el cerro Cuzco, Departamento de Potosí, Bolivia. Memoria Americana 21 (1):93-120.

Elíade, M. 1994. Lo Sagrado y lo Profano. Labor, Barcelona.

González, C. 2017. Arqueología vial del Qhapaq Ñan en Sudamérica: Análisis teórico, conceptos y definiciones. Boletín del Museo Chileno de Arte Precolombino 22 (1):15-34.

Hyslop, J. 1992. Qhapaqñan. El Sistema Vial Inkaico. Instituto de Estudios Andinos, Lima.

Hyslop, J. 1984. The Inka Road System. Academic Press, Orlando.

Ibacache, S. y G. Cantarutti R. 2006. Nuevas investigaciones en el Cerro Peladeros: Una huaca del Periodo Incaico en la cordillera de Chile Central. Revista Werken 10:63-79. 
Ibacache, S., G., Cantarutti, J. Berenguer y D. Salazar S. 2016. Adoratorios de altura y dominación incaica en el Alto Loa, norte de Chile. Intersecciones en Antropología 17:173-186.

Jacob, C. e I. Leibowicz 2011. Montañas sagradas en los confines imperiales. Nevado de Cachi, Salta-Argentina. Haucaypata 2:71-90

Lumbreras, L. 2006. Presentación. En El Qhapaq Nan-Camino Principal Andino. Plan de Acción Regional para un Proceso de Integración y Cooperación. Banco Interamericano de Desarrollo, Lima.

Lynch, T. 1995-1996. Inka roads in the Atacama: Effects of later use by mounted travellers. Diálogo Andino 14/15:187-203.

Martín, S. 2005. Caminos incaicos principales y secundarios en la Sa. de Famatina. La Rioja-Argentina. Actualización y revisión conceptual. Xama 15-18:21-35.

Martín, S. 2015. Rocas del Qhapaq Ñan: wankas y mojones en los caminos duales a las cumbres sagradas de la Sierra de Famatina (La Rioja-Argentina). Haucaypata. Investigaciones Arqueológicas del Tahuantinsuyo 10:78-97.

Martín, S. 2017. El Qhapaq Ñan en Famatina (La Rioja-Argentina): Ritualidad y manifestaciones sagradas Inkas en una Wak'a interregional al sur del Kollasuyu. Boletín del Museo Chileno de Arte Precolombino 22 (2):181-198.

Meyers, A. 2002. Los incas: ¿bárbaros advenedizos o herederos de Tiahuanaco? En El Hombre y los Andes. Homenaje a Franklin Pease, editado por J. Flores y R. Varón T. II, pp. 525-35. Pontificia Universidad Católica del Perú, Lima.

Mignone, P. 2010. Ritualidad estatal, capacocha y actores sociales locales. El cementerio del volcán Llullaillaco. Estudios Atacameños Arqueología y Antropología Surandinas 40:43-62.

Moyano, R. 2009. El adoratorio del cerro El Potro: Arqueología de alta montaña en la cordillera de Copiapó, norte de Chile. Estudios Atacameños 38:39-54.

Nielsen, A., J. Berenguer y C. Sanhueza 2006. El Qhapaqñan entre Atacama y Lípez. Intersecciones en Antropología 7:217-234.

Niemeyer, H. y M. Rivera 1983. Un camino inca en el despoblado de Atacama. Boletín de Prehistoria de Chile 9:91-193.

Núñez P. 1981. El camino del Inca. Revista CRECES 2:49-57.

Raffino, R. 1981. Los Inkas del Kollasuyu. Editorial Ramos Americana, La Plata.

Rebitsch, M. 1966. Santuarios en altas cumbres de la Puna de Atacama. Anales de Arqueología y Etnología 21:51-80.

Reinhard, J. 1983. Las Montañas sagradas: Un estudio etnoarqueológico de ruinas en las altas cumbres andinas. Cuadernos de Historia 3:27-62.

Reinhard, J. 1993-1994. Llullaillaco: Investigación del yacimiento arqueológico más alto del mundo. Anales de Arqueología y Etnología 48-49:105-129.

Reinhard, J. y C. Ceruti 2000. Investigaciones Arqueológicas en el Volcán Llullaillaco: Complejo Ceremonial Incaico de Alta Montaña. Salta, Argentina. Ediciones Universidad Católica de Salta, Salta.
Salazar, D., J. Berenguer y G. Vega 2013. Paisajes minero-metalúrgicos incaicos en Atacama y el altiplano sur de Tarapacá. Chungara Revista de Antropología Chilena 45 (1):83-103.

Schobinger, J. 1986. La red de Santuarios de Alta Montaña en el Cuntisuyu y el Collasuyu: Evolución general y Problemas interpretativos. Córdoba, Argentina. Comechingonia Número Especial pp. $297-$ 317.

Schobinger, J. 1998. Arqueología de alta montaña: Santuarios incaicos en los andes centro-meridionales. Beiträge zur Allgemeinen und Vergleichenden Archäologie, 18:363-399.

Schobinger, J. 2008. En la búsqueda del trasfondo cultural. Breve noticia sobre dos expediciones posteriores al extremo Norte de San Juan. En La Momia del Cerro El Toro. Segunda edición, corregida y ampliada, editada por J. Schobinger, pp. 15-62 Colección Cumbre Andina. Editorial de la Facultad de Filosofía y Letras. Universidad Nacional de Cuyo, Cuyo.

Vitry, C. 1997. Arqueología de Alta Montaña. Argentina. Yachayruna Revista de Divulgación Científica 1 (1):16-23.

Vitry, C. 2000. Aportes para el Estudio de Caminos incaicos. Tramo Morohuasi-Incahuasi. Salta, Argentina. Gofica, Salta.

Vitry, C. 2002. Apachetas y mojones, marcadores espaciales del paisaje prehispánico. Revista Escuela de Historia 1 (1):177-191.

Vitry, C. 2005. Propuesta metodológica para el registro de caminos con componentes inkas. Revista Andes 15:213-250.

Vitry, C. 2006. Arqueología del Nevado de Chañi. En Nevado de Chañi, editado por E. González y C. Vitry, pp. 31-70 Ed. Artes Gráficas, Salta.

Vitry, C. 2007. Caminos rituales y montañas sagradas. Estudio de la vialidad inka en el Nevado de Chañi, Argentina. Boletín del Museo Chileno de Arte Precolombino 12 (2):69-84.

Vitry, C. 2008. Los espacios rituales en las montañas donde los inkas practicaron sacrificios humanos. En Paisagens Culturais. Contrastes Sul-Americanos, editado por C. Terra y R. Andrade, pp. 47- 65, Escola de Belas Artes, Universidade Federal do Rio de Janeiro, Rio de Janeiro.

Vitry, C. 2009. Contribución al estudio de caminos de sitios arqueológicos de altura. Volcán Llullaillaco (6739 m). Salta-Argentina. Problemáticas de la Arqueología Contemporánea. Actas PostCongreso del XV Congreso Nacional de Arqueología Argentina, Tomo III, compilado por A. Austral y M. Tamagnini, pp. 127-140. Editorial de la Universidad Nacional de Río Cuarto, Río Cuarto.

Vitry, C. 2014. Los calzados utilizados por los incas para la alta montaña. Revista Haucaypata. Investigaciones Arqueológicas del Tahuantinsuyo 3 (8):91-109.

Vitry, C. 2015. Los caminos rituales del volcán Llullaillaco, Argentina (6739 msnm). Revista Haucaypata. Investigaciones Arqueológicas del Tahuantinsuyo 4 (10):65-77.

Vitry, C. 2017. El rol del Qhapaq Nan y los Apus en la expansión del Tawantinsuyu. Boletín del Museo Chileno de Arte Precolombino 22 (1):35-49. 
